\title{
A Brief Survey on Molecular and Electromagnetic Communications in Nano-Networks
}

\author{
Negar Rikhtegar \\ Department of Computer Engineering and \\ Information Technology \\ Shiraz University of Technology, Shiraz, Iran
}

\author{
Manijeh Keshtgary \\ Department of Computer Engineering and \\ Information Technology \\ Shiraz University of Technology, Shiraz, Iran
}

\begin{abstract}
Recent advancement in physics and nanotechnology have paved the way for manufacturing of processor, memory, batteries, transceiver, antenna and sensing units at nano-scale. A nanomachine is an integrated device with dimensions in nano-scale, and able to do simple tasks. By networking of nano-machines, they are able to perform the more complicated tasks by the cooperative manner and can play an important role in applications such as biomedical, environmental monitoring, industrial and military. Many novel nano-scale communication options have been currently proposed. However, there are four main nano-scale communication techniques: nanomechanical, acoustic, chemical or molecular and electromagnetic communications. However, this paper focuses on the molecular and electromagnetic communications as the promising nanoscale communication approaches, and then will be reviewed the novel nano-scale communication paradigms that are currently presented.
\end{abstract}

\section{General Terms}

Nano Network, Nano communication, Molecular communication, Electromagnetic communication

\section{Keywords}

Nano machine, Nano communication, Nano network, Nano sensor, Molecular communication, Electromagnetic communication

\section{INTRODUCTION}

A nano-machine is an integrated device around 10-100 $\mu \mathrm{m} 2$ in size and able to do simple tasks such as sensing, plain computation, communication and local actuation [6]. A nanonetwork is formed by connecting nano-machines; therefore it is able to perform more complicated tasks such as drug delivery, health monitoring and detection of biological or chemical attacks in nano-scale environments by cooperation of nanomachines [8]. By networking of nano-machines, they can carry out macro-scale objectives and work over a larger area, while a single nano-machine can only act at nano-level and closely its workspace. A nano-network makes an infrastructure to broadcast information among nano-machines in a large area, on the other hand, it will facilitate the interaction between nanonodes such as activation/deactivation, configuration of parameter, data receiving and actuation commands [1]. Due to small size and limited capabilities of nano-machines, traditional communication mechanisms are inapplicable in nano-networks [2]. Many novel nano-scale communication approaches are currently proposed. In general, there are four main techniques in nano-scale communication: nanomechanical, acoustic, chemical or molecular and electromagnetic communications [8]. In nanomechanical communication, the information is transmitted by a mechanical contact between transmitter and receiver. An acoustic communication is defined as the transmission of information through acoustic energy such as pressure variations. In molecular communication, the information is encoded in molecules which move as carriers from the transmitter nano-machine to receiver nano-machine. Electromagnetic communication is based on the modulation and demodulation of electromagnetic waves using components that are made based on novel nano materials $[1,2,8]$. In acoustic communication, the traditional acoustic transducers and radio frequency transceivers cannot be integrated at a nano-scale device because of their size and communication principle. Moreover, in nanomechanical communication, a physical and direct contact is needed between transmitter and receiver nanomachines. Therefore, the molecular and electromagnetic communications are the most promising approaches for nanonetworking [1]. This paper focuses on the molecular and electromagnetic communications as most studied nano-scale communication approaches and then for each one of them, we will review the novel nano-scale communication paradigms that are currently presented.

The rest of paper is organized as follows. In section 2, the approaches to the development of nano-machine are expressed. In section 3, the types of molecular communication in nanonetwork and their challenges are presented. In section 4, the electromagnetic communication in nano-network, concepts and architecture of nano-sensors, terahertz channel properties and electromagnetic communication challenges will be reviewed. In section 5, the applications of molecular and electromagnetic communication are presented. Finally, section 6 concludes the paper.

\section{DEVELOPMENT OF NANO- MACHINES}

Nano-machine can be artificially made from nanomaterials or naturally found in biological systems such as biological cell. There are three approaches for the development of nanomachines: top-down, bottom-up and bio-inspired approaches. Top-down approach is defined as downscaling of microelectronic and micro-electro-mechanical devices to develop a nano-machine. For this goal, techniques such as electron beam lithography and micro-contact printing are used. The resulting nano-machine keeps the architecture of microscale components that they are manufactured from. In the bottom-up approach, the nano-machines are developed by arranging the molecules unit together as molecular building blocks. But the technologies that can manufacture the molecular nano-machines by this approach do not exist yet. The third approach for the development of new nano-machines or integrating them into a more complex system such as nanorobots is bio-inspired. This approach is based on the features of future nano-machines that already exist in a living cell, which can be seen as a self-replicating collection of nano-machines [1, 2].The many of the biological nano-machines can be found in cells, including nano-biosensors, nano-actuators, biological data storing components, tools and control units [1]. Actually, the bio-inspired approach is inspired by biological nano-machines 
to create a model for development of new nano-machine. This network of nano-machines are based on molecular (chemical) signaling, that is used for inter-cell communication and leads to cooperation of multiple cells to perform a common objective $[1,2]$.

\section{NANO-COMMUNICATION BASED ON MOLECULES}

Molecular communication is defined as transmission and reception of desired information by means of molecules, and it can be used to interconnect nano-machines and forms a nanonetwork. Due to the size of molecular transceivers, the integration of them in nano-machines is feasible. These transceivers can react to specific molecules. Also, they can release specific molecules when an internal command is issued or some types of process are performed [6]. The released molecules can be propagated by one of the following methods: (a) Diffusion-based: the molecules are propagated by following spontaneous diffusion in a fluidic medium. (b) Flow-based: the molecules are propagated through a fluidic medium whose flow is guided. (c) Walkway-based: the molecules are propagated by active carriers which transport them through per-defined pathways [6].

In this communication, the transmitter nano-machine encodes the information in molecules and transmits them into the environment. The molecules move through the environment to the receiver nano-machine with a certain probability [4]. Fig.1 shows a scheme of a molecular nano-network with two nodes.

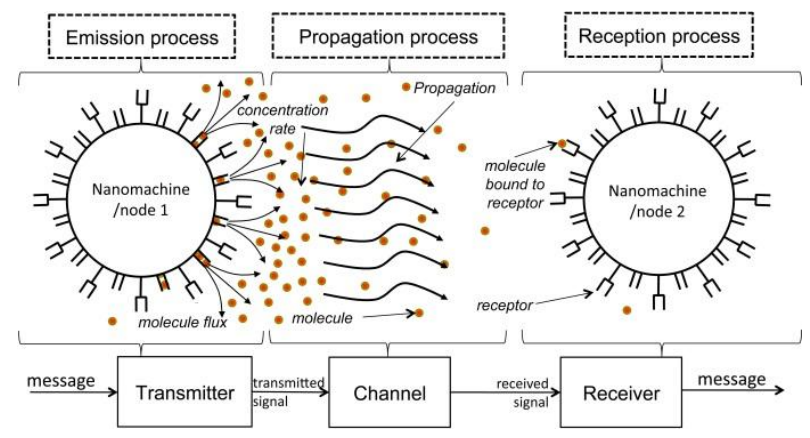

Fig 1: The scheme of two-node molecular communication [17]

Molecular communication uses active and passive transport methods to exchange information in the molecules. In passive method, information is randomly moved without using chemical energy and by forces in environment. It is clear that a high viscosity environment leads to slower diffusion of molecules. In fact, the molecules are flowed based on a concentration gradient. In active transport, the information is moved by chemical energy and consumes it to overcome its random motion in environment. This approach is independent of the concentration gradient. In this approach the information moves through mechanisms such as molecular motors or bacteria. For example, kinesin is a transport nano-machine in biological cell. It is a protein motor that moves along a microtubule. Microtubule is a polar protein filament that known as information pathways between sender and receiver $[4,7,12]$. Fig. 2 shows the active and passive transport methods.

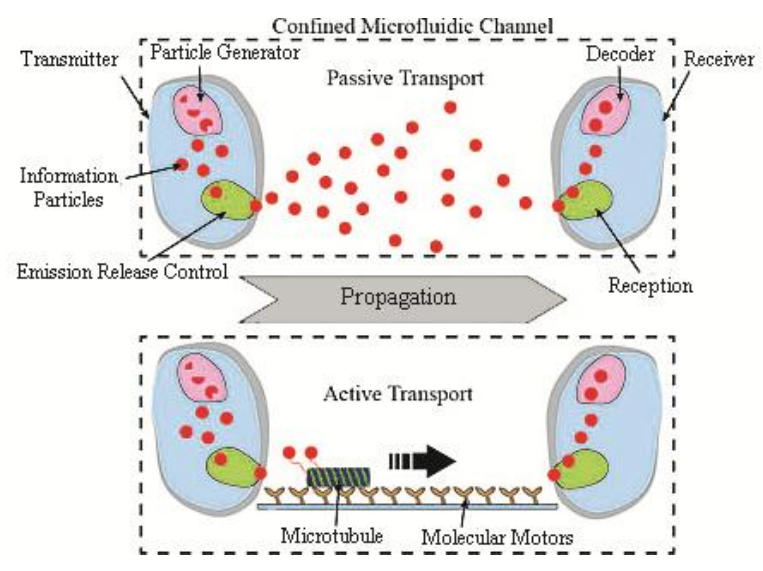

Fig 2: Active and passive transport methods [22]

\subsection{Molecular Communication versus Traditional Communication}

- In traditional communication, the information is encoded in electromagnetic, acoustic or optical signals. But in nanonetwork based on molecular communication, the information is encoded by means of molecules.

- The propagation speed of signal in traditional communication is much faster than the propagation speed of molecules in molecular nano-networks. In molecular communication, the information molecules physically move from the transmitter to the receiver, therefore, the environmental condition such as temperature can affect the propagation of the information molecules.

- The noise in traditional communication is additive noise, which is defined as an undesired signal overlapped with the information signals. But there are only two types of noise in molecular communication. First, it is additive noise that also occurs in traditional communication. This means another source release same molecules which are used to encode the message, therefore, the receiver may sense an incorrect concentration level. The second noise is undesired interaction occurring between information molecules and environmental molecules such as medium molecules.

- The traditional communication can transmit the text, voice and video over medium but in molecular communication, the message is a molecule.

- There are chemical processes in molecular communication. Therefore, their power consumption is low, while traditional communication consumes electrical power for their communication processes that is obtained from internal or external sources such as batteries or electro-magnetic induction [1].

\subsection{Encoding Technique}

Two types of coding techniques are introduced in nano-network based on molecular communication. The first one uses temporal concentration of specific molecules in the medium. According to the number of molecules per volume, the receptor decodes the received information. This is similar to transport of information by time-varying sequences in traditional networks. The second technique for encoding information uses internal parameters of the molecules such as chemical structure, relative positioning of molecular elements or polarization. However, for decoding of information, the receiver must be able to detect these specific molecules [1]. 


\subsection{Communication Range}

In molecular communication, the information molecules are transported between nano-machines within different distances. Three different approaches have been already proposed for molecular communication. They are short-range $(\mathrm{nm}-\mu \mathrm{m})$, medium-range $(\mu \mathrm{m}-\mathrm{mm})$ and large-range $(\mathrm{mm}-\mathrm{m})$ communications [4].

\subsubsection{Short-range communication}

This type of molecular communication takes place in range of $\mathrm{nm}$ to few $\mathrm{mm}$. The communication in biological nanonetworks such as intra-cell and inter-cell is a short-range communication, due to the size of living cells. There are two techniques for short-range communication, namely, the molecular motors and calcium signaling.

\subsubsection{Short-range communication: Molecular Motors}

Molecular motors are biological nano-machine for transporting vital particle among living organisms. Molecular motors such as Kinesin and Dynein are protein complexes that convert chemical energy to motion $[1,15]$. They are communication carrier for nano-machines within a short-range communication $[1,2]$. Molecular motors are moved by molecular rails named microtubules [1] as shown in fig.3.

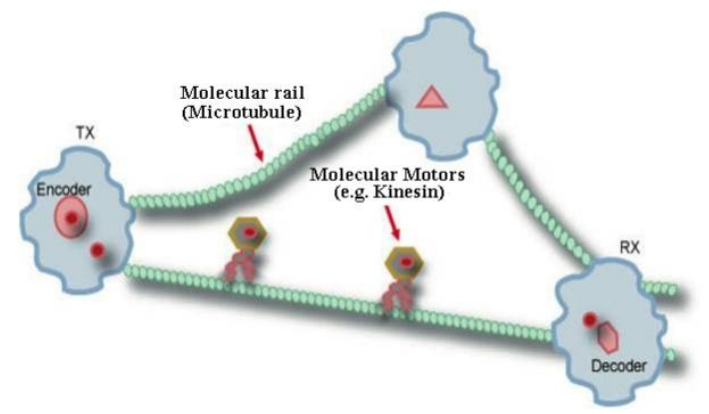

Fig 3: Molecular motors communication systems [1]

Microtubules are information pathways between sender and receiver nano-machines [4]. On the other hand, molecular motors and microtubules can be useful in the bio-hybrid communication between man-made nano-machine and biological molecular machines [1]. For example, in drug delivery application, the nano-machine can interact with cell organelles by help of these communication agents [1]. Before the communication process begins, the development of microtubules as network infrastructures is important so molecular motors can be moved. The microtubules are able to interconnect one nano-machine with others. According to network infrastructure, the transmitter nano-machine can select the unicasting or multicasting mechanisms. To achieve the unicasting, the sender selects the microtubule which is located between it and intended receiver. It is similar to point-to-point communication in traditional systems. To implement the multicasting mechanism, the sender releases several molecules that are self-assembled onto molecular motors which moving on different microtubules. Therefore, they reach to different destinations. The molecular motors move unidirectionally along microtubules. The polarity of microtubule determines the direction of molecular motors. For example, kinesin moves toward the + end and dynein tends to the - end of the microtubule. Therefore, the use of different molecular motors such as kinesin and dynein or a pair of opposite microtubule between two nano-machines can form a bidirectional communication link. The information in molecules can be encapsulated in vesicle [1]. A vesicle is a fluid or an air-filled cavity (like a bubble) that stores and transports cellular products or digests metabolic wastes within the cell. Molecular motors carry the vesicles from transmitter to the receiver.

The molecular communication based on molecular motors consists of the following tasks:

- Encoding: when a stimulus is applied to the transmitter, it generates information molecules. The encoding process consists of selecting proper molecules that are able to represent the information or control the desired reaction of the receiver.

- Transmission: the information packets can be propagated by means of the affinity between information molecules and molecular motors. Due to the high affinity between vesicles as biological capsules and molecular motors, the information molecules can be encoded in vesicles which are bonded to the molecular motors.

- Propagation: in this step, the information molecules travel along microtubules and follow the direction of them instead of moving randomly or diffusion propagation.

- Reception: when the molecular motor reaches to the receiver, it detaches information molecules from molecular motors. This process can be performed by the affinity between protein tags and the receptors at the receiver. The sender attaches protein tags to molecular motors that can be bonded to the specific receptors on the receiver. The extraction of information from vesicles is done by cellular processing such as fusion or pore formation.

- Decoding: a receiver nano-machine can have several receptors. Every receptor reacts to specific information molecules. According to the information molecules, the receiver selects a desired reaction [1].

\subsubsection{Short-range communication: Ion Signaling}

In molecular signaling technique, the information is transmitted by varying a given concentration of ions. The molecular concentration level is similar to the carrier in classical wireless communication [2]. Molecular singling includes different messengers such as ATP substance, calcium ions $(\mathrm{Ca}+2)$ or inositol 1,4,5-triphosphate $\left({ }^{I P_{3}}\right)$ [1]. The example of molecular signaling is calcium signaling among cells [2]. Calcium signaling is mostly used in inter-cell communication. It is responsible for exchange information among cells to perform many tasks at a coordinated manner. Chemosignal pathway is defined as sequential propagation of the chemosignal by different messengers. In other words, the information which was carried by a messenger, in a certain point of pathway, is transferred to another messenger. This process continues until the information reaches its destination. This multi-messenger propagation makes the signal to be amplified at different points of the pathway where the messenger is replaced. This makes the different decoding results as well as the propagation parameters could be adapted to a specific environment. The calcium signaling is a more flexible approach than the communication based on molecular motors and microtubules that were previously explained [1]. Using calcium signaling, all of nanomachines that are located within the propagation radius of transmitter nano-machine can receive the message. This communication scheme is similar to the broadcast mechanism in traditional wireless communication. While in communication based on molecular motors, only one receiver can receive a message which is sent by a unique transmitter. According to the deployment of the nano-machines, the information can be transmitted by one of the following approaches: 
Direct access: if nano-machines are connected to each other and are located one next to each other. The calcium ions travel from one nano-machine to the next one through gap junctions as biological gates that allow ions or molecules to move between cells.

Indirect access: if there is no direct contact between nanomachines, the transmitter encodes the information using different concentration level of first messenger and releases them to the medium. The information through a diffusion process will be reached to the receiver nano-machine. Then, they will generate a calcium signal inside the receiver. Fig. 4 shows signal propagation in calcium signaling by gap junctions and diffusion mechanisms.

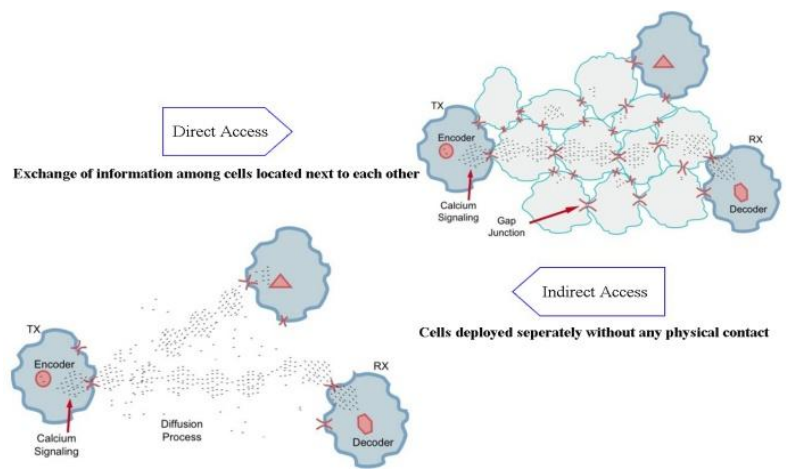

Fig 4: Signal propagation in calcium signaling communication [19]

In indirect access, the principle of affinity between ligand and receptor is a key process. A ligand is a molecule that can bind to a protein such as receptor. The ligand (i.e. information molecules) binds to a receptor which is located on the external membrane of the receiver. These receptors convert this binding to an internal signal such as $\mathrm{Ca}^{+2}$ signal in cell which can be decoded by cell components. Therefore, the ligand is known as the first messengers that transfer the information outside the cell and the internal signal molecules are the second messenger that moves the information inside the cells.

In direct access, the signal moves by second messenger such as $I P_{3}$, among cells. $I P_{3}$ is a messenger that can excite the cell organelles such as endoplasmic reticulum to releases calcium ions as shown in fig.5.Therefore, the propagation of IP3 by gap junctions leads to propagation of $\mathrm{Ca}+2$ wave among the cells

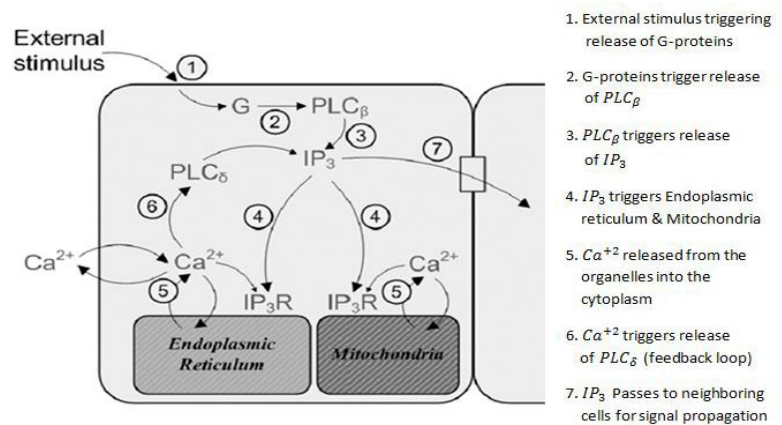

Fig 5: The process of releasing calcium ions in cell [16]

In general, a signaling network connects transmitter and receiver nano-machines to each other by interconnecting nodes and then the information is propagated by calcium signal. There are challenges in communication based on calcium signaling. $\mathrm{Ca}+2$ ions can be easily affected the charged particles in the medium. Thus, the channel is not noise-free. On the other hand, the calcium signaling may interfere with biological processes such as muscle contractions or neuro signals [1]. The communication based on calcium signaling consists of the following steps:

- Encoding: in direct access, the sender encodes information using $\mathrm{Ca}+2$ that can be modulated in frequency (FM) or amplitude (AM). But in indirect access, the sender must encode the information in molecules as first messenger. Also, the first messenger can be modulated by FM or AM schemes. As shown in fig.5, an external stimulus can lead to release of $\mathrm{Ca}+2$.

- Transmission: in direct access, the transmitter nano-machine stimulates neighboring nano-machines and then the propagation of $\mathrm{Ca}^{+2}$ ions is started. In indirect access, the transmitter initiates the signaling process by releasing substances to environment.

- Signal propagation: in direct access, the moving of ${ }^{I P_{3}}$ among neighboring cells lead to release $\mathrm{Ca}+2$ from $\mathrm{Ca}+2$ stores such as endoplasmic reticulum which excited by ${ }^{I P_{3}}$ as shown in step 4 of fig.5. The diffusion of ${ }^{I P_{3}}$ among other nanomachines makes the $\mathrm{Ca}+2$ wave propagation across nanonetwork. In indirect access, the first messenger propagates by diffusion model. When the information molecules bind to the receptors of receiver, they convert them to $\mathrm{Ca}+2$ signals.

- Reception: in direct access, the receiver detects the $\mathrm{Ca}+2$ concentration using gap junction with neighbors. When the receiver receives a message, it closes its gap junction, and thus the ${ }^{I P_{3}}$ propagation is stopped. In indirect access, the receptors of the receiver nano-machine detect the received information molecules. The different information molecules can be detected by different receptors. Therefore, a parallel communication channel among several nano-machines can be formed.

- Decoding: the receiver reacts to $\mathrm{Ca}+2$ concentration. In direct access, this concentration depends on the value of the $I P_{3}$ influx and the $\mathrm{Ca}+2$ that is received by the receiver. In indirect access, it depends on the bindings between receptors and information molecules [1].

\subsubsection{Medium-range communication}

In short-range molecular communication such as molecular signaling, the transmission delay of a particle in a certain distance, increases quadratically with distance. Thus, the existing short-range molecular communication schemes are not effective for the distances longer than a few $\mu \mathrm{m}$. Therefore, the medium-range molecular communication is proposed for distances from $\mu \mathrm{m}$ to $\mathrm{mm}$ and two new communication techniques, flagellated bacteria and catalytic nanomotors are introduced to support this type of communication. Both methods encode the information in DNA sequences. In this type of communication, the nano-machines will be able to communicate by DNA nucleotides, including Adenine, Thymine, Cytosine and Guanine (A, T, C, G). The transmitter nano-machine inserts the information into a set of DNA base pairs that called DNA packet. The whole DNA sequence is encapsulated in a vesicle and is transported by short-range communication to the transmitter gateway. When the information reaches to the transmitter gateway, it will identify 
the location of receiver's gateway. If the receiver nano-machine is located at the transmitter gateway domain, it will send the DNA packet by using the point-to-point link to the receiver. Otherwise, the gateway will multiplex the packet and will send it by medium-range communication to the receiver's gateway [2].

\subsubsection{Medium-range communication: Flagellated}

\section{Bacteria}

The flagellated bacteria can consume chemical energy and convert it to motion. Among all possible flagellated bacteria the E.coli (Escherichia coli) is the best choice because its genome sequence is well known. Only one circular DNA exits in its nucleoid and there are the smaller DNA sequences in its cytoplasm by a circular arrangement. These DNA circles are responsible for exchanging of genetic materials among bacteria cells. E.coli is used to carry DNA messages. First, a specific mutant of the bacteria is selected that can only react to the specific attractant particles which is released by receiver to the environment. Second, the DNA message is located inside the bacterium cytoplasm. Finally, the bacterium is released to the environment and depends on its instincts, it moves to the intended receiver using attractants that are continuously released $[2,13]$. The communication process using flagellated bacteria is performed by the following steps:

- Encoding: In this process, the DNA packet is located inside bacteria's cytoplasm, by using genetic engineering procedures. This process consists of three steps that are shown in fig.7. As can be seen in this figure, the encoding process includes of (i) the cleaving of plasmid phase in restriction sites (ii) linking of DNA packet (containing the information) to the plasmid (iii) inserting plasmid inside bacteria's cytoplasm. Since each bacterium is resistant to a certain antibiotic, a specific antibiotic can be applied to a group of bacteria and thus the correct bacterium can be chosen, which contains the desired DNA information.

- Propagation: a number of receptor are located around the bacteria membrane and can sense the environment to find attractant particles and moves towards them, where there is the best condition for living. This process is called chemotaxis. E.coli moves in runs or tumbles manner. In run, the flagella motors spin counterclockwise and the bacterium moves in a straight line. Whereas, in small tumble period, the independent motion of several flagella on the cell in clockwise cause it to tumble randomly. Fig.6 shows the patterns of E.coli movement. In run period, the bacteria can detect the nutrients (sugars, amino acids) concentration level several times by using chemoreceptors on the cell membrane. During running period, if the bacteria sense that a concentration is increasing, the running time becomes longer. This bias makes the ability for cells to find the places where the environmental conditions are better.
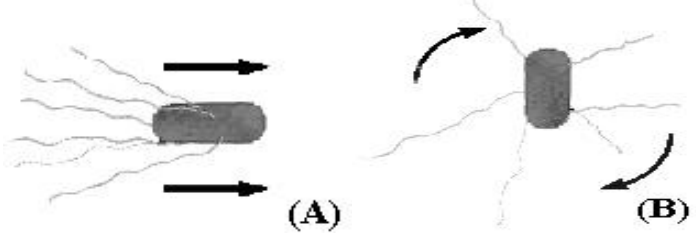

Fig 6: runs (A) and tumbles (B) periods [23]

- Reception and decoding: in this step, when a plasmid reaches to the receiver, the DNA packet must be extracted from it. This process is done through cleave plasmid in restriction sites by restriction endonucleases enzymes. This allows the receiver to isolate the DNA packet from the plasmid which is cleaved and then process it $[2,13]$.

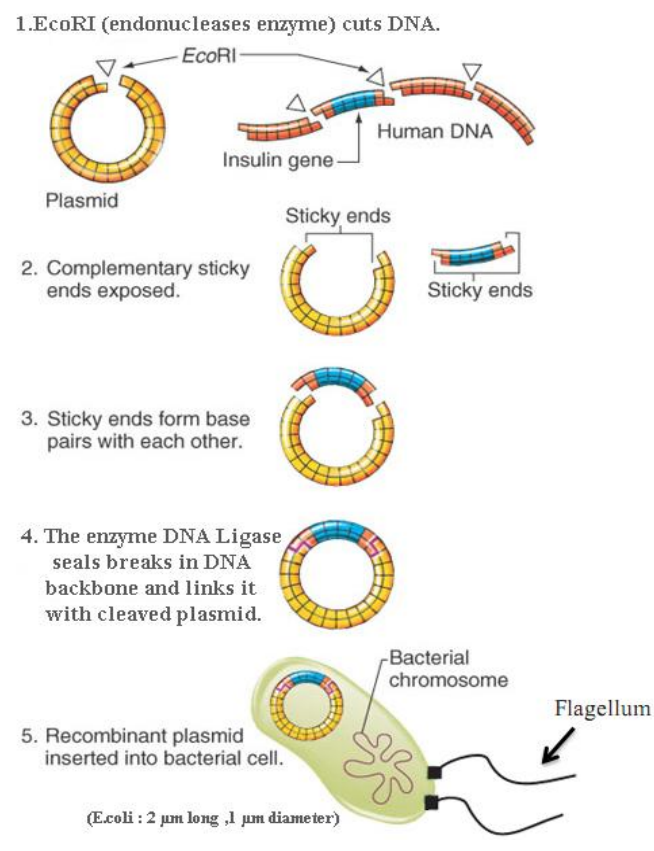

Fig 7: The process of encoding of the DNA packet using plasmids

\subsubsection{Medium-range communication: Catalytic Nanomotors}

Catalytic nanomotors are synthetic particles that can propel themselves and other small objects through self-generated gradients. The catalyzing of the free chemical energy in the environment forms these gradients. Gold $(\mathrm{Au})$ and platinum $(\mathrm{Pt})$ nanorods are the most common types of catalytic nanomotors. These particles are used to transport the DNA information between gateways [2, 14]. The $\mathrm{Pt} / \mathrm{Au}$ nanorod moves autonomous and approximately in a unidirectional path, in aqueous hydrogen peroxide $\left(\mathrm{H}_{2} \mathrm{O}_{2}\right)$ solution by catalyzing oxygen $\left(\mathrm{O}_{2}\right)$ at the Platinum end. The Platinum acts as a catalyst for decomposition of $\left(\mathrm{H}_{2} \mathrm{O}_{2}\right)$. The movement of $\mathrm{Pt} / \mathrm{Au}$ in $\left(\mathrm{H}_{2} \mathrm{O}_{2}\right)$ is autonomous while a directed motion is needed, so they can be useful for many applications as shown infig.8 (A).By adding Nickel segment, the direction of nanorod can be controlled. Due to the ferromagnetic property of Nickel, it can be magnetized and control the direction of nanorod in the presence of magnetic field. The $\mathrm{Au} / \mathrm{Ni} / \mathrm{Au} / \mathrm{Ni} / \mathrm{Pt}$ striped Nanorod is directed when is exposed by an external magnetic field $[2,15]$ as shown in fig.8 (B). 


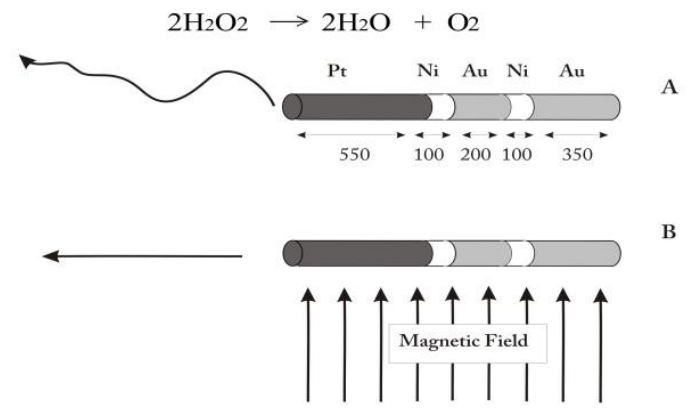

Fig 8: The movement of $\mathrm{Pt} / \mathrm{Ni} / \mathrm{Au} / \mathrm{Ni} / \mathrm{Au}$ rods in hydrogen peroxide without the magnetic field (A) and with an applied magnetic field (B) [2]

This type of communication includes the following steps:

- Encoding: in this step the plasmids containing DNA packet are attached on the catalytic nanorod surface. First, the catalytic nanorod is inserted into the AEDP (3-[(2aminoethyl)dithio]propionic acid $\bullet \mathrm{HCL})$ solution. AEDP binds to Nickel segments. As a result, the plasmids which are conjugated with the AEDP attaches to Nickel surface. In order to compressing and immobilizing the plasmids, the nanorod is inserted into a $\mathrm{CaCl}_{2}$ solution. Finally, the transferrin protein that has been attached to Gold segment helps to the receptors which are located on the cellular membrane to detect the rod. In this case, the plasmid can be encoded on the Nickel surface (4.1012 molecules.cm-2). The fig. 9 shows this process [2].

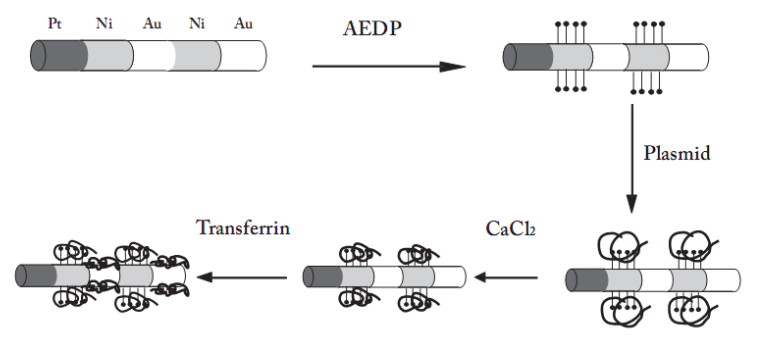

Fig9: Encoding of the plasmids in the $\mathrm{Au} / \mathrm{Ni} / \mathrm{Au} / \mathrm{Ni} / \mathrm{Ptnanorod}[2]$

Propagation: Two different solutions are used in order to the plasmid can reach to the proper receiver. In first option, the nanorods are guided by pre-established magnetic paths from the emitter to the receiver. The second is the making of a raft by joining of several nanorods together and use of the chemotactic process. The raft is covered by inhibitor particles of a solution. These particles bind to the receptors which is located on rods surface. Since the difference of concentration gradient of the inhibitor particles between one corner of the raft that is closer to the inhibitor source (moving slower) and the other corner that is farther the inhibitor, the raft will move towards the inhibitor source (S). Fig.10 shows this chemotactic process.

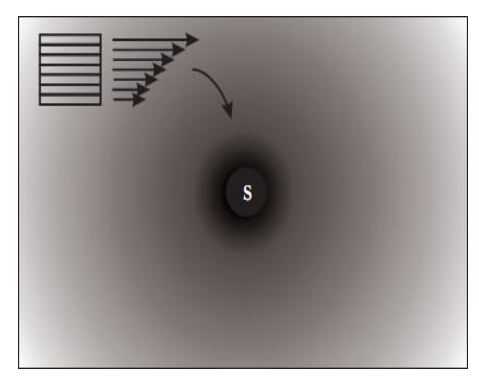

Fig10: Chemotactic process of a raft [2]

The challenge of first solution is that a magnetic field must be applied and controlled. On the other hand, when there are several different communication channels, it is not feasible to handle them due to the scale of nanorods. The challenge of second solution is that the velocity of the raft by moving toward to inhibitor source is decreased. The raft can transmit a much more information than a single nanorod because of its larger size [2].

- Reception and decoding: this step is performed by a transferrin protein that has been attached to the gold segment of the rod. This protein is used to deliver the iron ions to the cells of the body. It can bind with the transferrin receptors which are placed over the cell membrane and goes inside the cells, and then the DNA packet is extracted from plasmid. Hence, the gateway must include membrane receptor to uptake the nanorods [2].

\subsubsection{Long-range communication: Pheromones}

The long-range communication is defined as the communication process with the transmission distance of millimeter up to meter $[1,5]$. This type of communication is also seen in nature. In nature, many species release pheromones that only the other member of the same species can identify them [2]. The pheromones can be considered as molecular messages containing information that can only be decoded by specific receiver nano-machine which are equipped with corresponding decoder and can react to them [1, 2]. The advantage of communication based on pheromone is that they can act in dry and wet environment. The information in this network is just encoded on the molecules. Since a message consists of molecules, therefore for exchanging and encoding information, many different combinations can be used. The reception process is based on the receptors that are located on the receiver nano-machine. They can be considered as the receiver antenna or transducer, which transform the energy of the message into a proper reaction at the receiver. The communication based on pheromone is a scalable communication system $[1,5]$. However, there are no artificial nano-machines that can execute the long-range communication. The long-range communication based on pheromone includes the following steps:

- Encoding: in this step, the pheromones are selected for transmission of information and invoke the proper reaction at the receiver.

- Transmission: in this phase, the pheromones that are selected in previous step are release to medium. The transmitter transmits the information through liquids or gases by a voluntary manner.

- Propagation: in this step, the pheromones are propagated from the transmitter to the receiver nano-machines by diffusion model. In biological systems, by measuring the 
concentration level of pheromones near the receiver, it can receive the pheromone message.

- Reception: Once the Pheromone reaches to the receiver, the receptors on the receiver can detach the information molecules from it. These receptors are proteins, which can bind to pheromone through the high affinity force that exists between them. This process is based on ligandreceptor binding principle, similar to the communication based on calcium signaling.

- Decoding: in this step, the receiver reacts to the received information [1]
Some types of molecular communication were described in previous sections. Table.1 shows characteristics of these molecular communications in summary.

\subsection{Examples of Novel Schemes in Molecular Communication}

Balasubramaniam et al. in [3] have proposed the use of neuronal networks for molecular communication (use of neurons as a networking component). First, they design an interface between nano-devices and neurons for signal initiating. Second, they present a transmission scheduling to ensure that the several signals which are simultaneously transmitted, with minimum interference will reach the receiver. The solution for this scheduling scheme is based on the generic algorithm optimization technique.

Table 1. The characteristics of molecular communications

\begin{tabular}{|c|c|c|c|c|c|c|}
\hline $\begin{array}{c}\text { Communication } \\
\text { Range }\end{array}$ & Carrier & Encoding & Transmission & Propagation & Reception & Decoding \\
\hline \multirow[t]{2}{*}{$\begin{array}{l}\text { Short-range } \\
\text { (nm to } \mu \mathrm{m})\end{array}$} & $\begin{array}{l}\text { Molecular } \\
\text { Motor \& } \\
\text { microtubule } \\
\text { (wired) }\end{array}$ & $\begin{array}{l}\text { selecting proper } \\
\text { molecules that } \\
\text { are able to } \\
\text { represent the } \\
\text { information }\end{array}$ & $\begin{array}{l}\text { Attach the } \\
\text { information } \\
\text { packet to the } \\
\text { molecular motor }\end{array}$ & $\begin{array}{c}\text { information } \\
\text { molecules travel } \\
\text { along } \\
\text { microtubules and } \\
\text { follow the } \\
\text { direction of them }\end{array}$ & $\begin{array}{l}\text { Detaching of } \\
\text { information } \\
\text { molecules from } \\
\text { molecular } \\
\text { motors }\end{array}$ & $\begin{array}{l}\text { According to } \\
\text { information } \\
\text { molecules, the } \\
\text { receiver selects } \\
\text { a desired } \\
\text { reaction }\end{array}$ \\
\hline & $\begin{array}{l}\text { Ion (e.g., } \\
\text { calcium) } \\
\text { Signaling } \\
\text { (wireless) }\end{array}$ & $\begin{array}{l}\text { Information is } \\
\text { encoded in } \mathrm{Ca}^{2+}\end{array}$ & $\begin{array}{l}\text { Initiates the } \\
\text { signaling } \\
\text { process }\end{array}$ & $\begin{array}{l}\text { Propagation of } \\
\text { the } \mathrm{Ca}^{2+} \text { waves }\end{array}$ & $\begin{array}{c}\text { Receiver } \\
\text { perceives the } \\
\mathrm{Ca}^{2+} \\
\text { concentration }\end{array}$ & $\begin{array}{l}\text { Receiver reacts } \\
\text { to the } \mathrm{Ca}^{2+} \\
\text { concentration }\end{array}$ \\
\hline \multirow[b]{2}{*}{$\begin{array}{l}\text { Medium-range } \\
\text { ( } \mu \mathrm{m} \text { to } \mathrm{mm})\end{array}$} & $\begin{array}{l}\text { Flagellated } \\
\text { Bacteria } \\
\text { (wireless) }\end{array}$ & $\begin{array}{l}\text { DNA packet is } \\
\text { inserted inside } \\
\text { the bacteria's } \\
\text { cytoplasm }\end{array}$ & $\begin{array}{l}\text { Bacteria sense } \\
\text { gradients of } \\
\text { attractant } \\
\text { particles }\end{array}$ & $\begin{array}{l}\text { Bacteria moves } \\
\text { towards attractant } \\
\text { particles } \\
\text { (chemotaxis). }\end{array}$ & $\begin{array}{l}\text { The receiver } \\
\text { releases } \\
\text { attractants so } \\
\text { the bacteria } \\
\text { can reach it. }\end{array}$ & $\begin{array}{l}\text { DNA packet is } \\
\text { extracted from } \\
\text { the plasmid }\end{array}$ \\
\hline & $\begin{array}{l}\text { Catalytic } \\
\text { Nanorod } \\
\text { (wireless) }\end{array}$ & $\begin{array}{l}\text { The DNA } \\
\text { plasmids attach } \\
\text { on the catalytic } \\
\text { nanorod surface }\end{array}$ & $\begin{array}{l}\text { Plasmid can be } \\
\text { transmitted by } \\
\text { Single nanorod } \\
\text { Or } \\
\text { raft ( made by } \\
\text { joining of } \\
\text { several } \\
\text { nanorods) }\end{array}$ & $\begin{array}{c}\text { Nanorods moves } \\
\text { by Using } \\
\text { Magnetic Fields } \\
\text { (single nanorod) } \\
\text { Or } \\
\text { concentration } \\
\text { gradient of the } \\
\text { inhibitor (raft) }\end{array}$ & $\begin{array}{l}\text { Binding } \\
\text { nanorod } \\
\text { transferrin and } \\
\text { transferrin } \\
\text { receptor on the } \\
\text { receiver }\end{array}$ & $\begin{array}{l}\text { DNA packet is } \\
\text { extracted from } \\
\text { the plasmid }\end{array}$ \\
\hline $\begin{array}{c}\text { Long-range } \\
\text { (mm to } \mathbf{m})\end{array}$ & $\begin{array}{c}\text { Pheromones } \\
\text { (wireless) }\end{array}$ & $\begin{array}{l}\text { Selection of the } \\
\text { specific } \\
\text { pheromones to } \\
\text { transmit the } \\
\text { information }\end{array}$ & $\begin{array}{l}\text { the pheromones } \\
\text { release to } \\
\text { medium }\end{array}$ & $\begin{array}{l}\text { pheromones are } \\
\text { propagated into } \\
\text { the medium by } \\
\text { diffusion model }\end{array}$ & $\begin{array}{l}\text { Pheromones } \\
\text { bind to the } \\
\text { Receptor }\end{array}$ & $\begin{array}{l}\text { Interpretation } \\
\text { of the } \\
\text { information } \\
\text { and react to } \\
\text { them by } \\
\text { receiver }\end{array}$ \\
\hline
\end{tabular}

The first case makes the external device contact to neurons and switches the neurons to signal transmission. Once a device has switched and signaled the neuron, then the proposed scheduling scheme ensures that the signal is transmitted through the neuron network will reach to the destination with minimum interference. The neurons have self-organizing ability, and they can form a network through transmission of neurites (any projection from the cell body of a neuron). There are sensors that are interfaced to neurons. They locate in the places that the signaling is propagated to the receiver and activate neuron signaling. Therefore, there is a need for a sensor to emit an agent who can activate the signaling. In signaling initiation, if sensors emit randomly, this could lead to a large number of interferences in the neuron network. Therefore, a scheduling protocol is required to ensure that signals are received with minimum interferences. Finally, they propose a single bit-Time Division Multiplex Access (TDMA) scheduling. In this scheme, each of the neurons fire at a given time. There are two amplitudes for bit 0 and 1 , therefore, only one bit is transmitted per slot. After a neuron is fired, it waits for a refractory period (to $\mathrm{Ca} 2+$ returns to the intracellular stores). Other sensors can transmit in waiting time (parallel transmission). There are 
many differences between a neuron link and a wireline communication link including:

In most communication networks, each link has different bandwidth and is able to provide a number of flows, whereas in the neuron link, each link can only provide a finite number of capacities.

Once a neuron is fired, it waits for a refractory period. During this period, neuron cannot transmit any signal, while in wireline communication when the transmission of old flows was completed then the new flow can be immediately transmitted.

Enomoto et al. in [4] design a self-organizing microtubule network. Microtubules are protein filament that is naturally appeared in the aqueous environment of cells [4]. A microtubule network can interconnect many nano-machines together. Two approaches are proposed to form microtubule network at a self-organizing manner. Their first approach is based on the polymerization and depolymerization of microtubules. The second approach is based on molecular motors to reform a microtubule network. In general, the first approach makes the microtubules interconnect sender and receiver in a self-organizing manner. The second approach leads to reorganizing a microtubule network by molecular motors. The simplest case is when nano-machines are interconnected by there a single microtubule. When information molecules have multiple microtubules, molecular motors can bind to multiple close microtubules, and then they form a molecular motor complex (crosslink microtubule). However, the crosslinked microtubules can be used as a pathway to move the molecular motors. This is important to all the microtubules that are located in crosslinked microtubules to can be direct in the same orientation. If they do not have the same direction, this is caused the motors move along incorrect direction or not move at all. Another issue is to form a self-organizing microtubule network. The controlling of molecular positioning by human is impossible, because there are a large number of molecules in a nano-scale environment. The self-organizing microtubules can dynamically adapt themselves to the local changes [4]. In the beginning, the sender and receiver nano-machines are randomly distributed and there is no microtubule to interconnect the nano-machines. In this approach, the sender binds to the microtubules seeds, and receiver binds to the microtubule binding sites. A microtubule is created by nucleating in random directions that is started from the place of microtubule seeds of sender nano-machine. This random elongation is radially occurred in plus ends of microtubule, therefore, they are propagating away from the sender nanomachine while, the minus ends of the nucleating microtubules are fixed on the sender nano-machine. The elongation of microtubules continues until they bind to the cap sites of the receiver nano-machine. A microtubule network is formed by repeating the elongation and shrinkage of microtubules. At the end of this process, the microtubules are interconnected between sender and receiver nano-machines. Fig.11 shows the process of forming a microtubule network in a self-organizing manner.

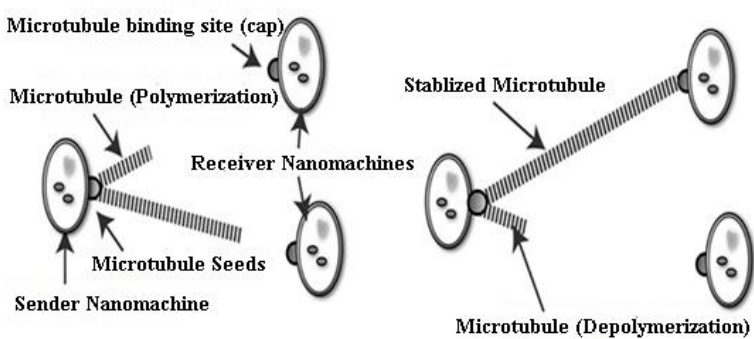

Fig 11: The formation of a microtubule network using polymerization and depolymerization of microtubules [4]

\subsection{Molecular Communication Challenges}

Besides the points mentioned above, we are faced with challenges in molecular communication including:

- How many new molecules are required to encode the information and diffusion through the network?

- How to control the binding process to release the information molecules into the medium

- How to manage the incoming information molecules.

- How to handle uncontrolled medium conditions such as rain, obstacles, wide that can affect the communication signal.

- The communication protocols to analyze and understand the channel model and signal propagation properties must be developed.

- A medium access protocol is needed to define channel access mechanism, fairly.

- In larger networks, for support of bidirectional and multihop communication, an addressing scheme is needed [1].

\section{ELECTROMAGNETIC COMMUNICATION}

As was explained earlier, the molecular communication is based on bio-molecules as a communication medium, while electromagnetic communication is based on communication using electromagnetic waves and wireless technology. Recent advancements in nanotechnology and carbon electronics have paved the way to produce electronic nano-device such as nano-memories, nano-processor, nano-batteries, and nanoantenna. The nano-particles show new behavior in nano-scale that cannot be observed at the microscopic level. The goal of nanotechnology is not miniaturization, but to create nanodevice with new functionality and unique characteristics such as nano-sensors $[6,3]$.

\subsection{The Nano-Material}

Due to the small size of nano-machine, wiring a large number of them is not feasible. On the other hand, the current electromagnetic transceivers cannot be easily integrated into the nano-devices because of their size and complexity. Hence, only the use of carbon structure may be able to develop the electronic nano-components $[6,9]$. One the most known nanocarbon structure that is referred it as the wonder material of the 21 st century is graphene. Graphene is a one-atom-thick planar sheet that consists of carbon atoms arranged on a honeycomb crystal lattice. Two of the known derivatives of graphene are Carbon Nanotubes (CNT) and Graphene Nanoribbon (GNR). The CNT is a folded nanoribbon. Furthermore, the GNR is a thin strip of grapheme as shown in 
fig.12. The carbon materials have the electrical and optical characteristics that are analyzed in light of quantum mechanics. These properties include of high current capacity and high thermal conductivity (it is 10 times better than copper) that makes them efficient in terms of energy. Their mechanical strength is extremely high (it is 100 times stronger than steel) and due to the special arrangement of carbon atoms, they have a very high sensitivity. These specifications make new opportunities for device-technologies such as nanobatteries, nano-memories, nano-processors, nano-antenna and nano-transceivers

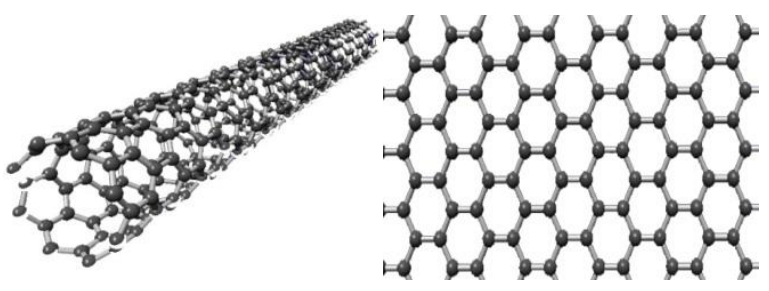

Fig 12: The scheme of CNT and GNR structure

\subsection{Nano-Sensor Architecture Based on Electromagnetic Communication}

The nano-sensors are very small devices that are able to do simple tasks such as sensing, simple computation or local actuation. A nano-sensor is composed of the following units:

- Sensing unit: is developed using the nanomaterial such as graphene and its derivatives such as CNTs and GNRs, due to their prominent sensing capabilities [6].

- Actuation unit: using this unit, the nano-sensor reacts to itself surrounding events. There are different types of actuators: physical and chemical. In Physical nanoactuator, a physical change of nanotube creates a change in the electrical properties. Applying an electrical wave can create a physical deformation in a nanotube. Chemical and biological nano-actuators work by interacting with nano-particles, electromagnetic fields and heat. For example, the cancer cells can be destroyed by nanoelectromagnetic particles [6].

- Power unit: the nano-batteries can be built by nanomaterials with high power density, good life-time and charge/decharge periods. However, the nano-sensor may be used in inaccessible places where there is no possibility to recharge. Therefore, the concept of self-power has been recently presented. The self-power devices can convert a form of energy (for example, mechanical, vibrational or hydraulic energies) into electrical energy. This conversion is performed by the piezoelectric effect of zinc oxide ( $\mathrm{ZnO}$ ). Fig.13 shows the piezoelectric effect of $\mathrm{ZnO}$. Therefore, the nanowires can be built by zinc oxide. So, when the nanowires are bent, an electrical voltage is generated in nanowire. Moreover, the energy of the electromagnetic wave can be useful, for example, a resonator can transform electromagnetic wave into vibrational energy, and then the $\mathrm{ZnO}$ nanowires convert it to electrical energy. Another source would be the use of chemical batteries based on ATP (Adenosine Triphosphate) which can harvest energy from chemical reactions inside cells or living organisms.

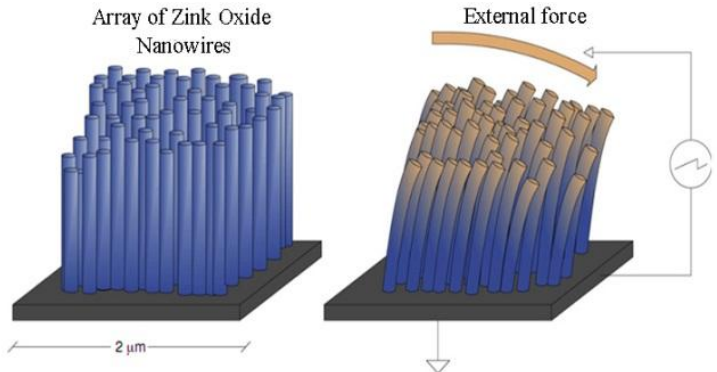

Fig13: The piezoelectric effect of $\mathrm{ZnO}$ [6]

- Processing unit: nano-processors are built by graphenebased transistors. Graphene makes ballistic transport of electrons that allows developing faster switching devices. For instance, the world smallest transistor has been developed by physics group at the University of Manchester in 2008. It is based on a thin strip of graphene just 1 atom $\times 10$ atoms [20]. However, the number of transistors in a nano-processor and the complexity of the operations are limited, due to the small size of nanosensors.

- Storage unit: nano-memories store a single bit on a single atom by nano-material and new technologies. The atomic memories have been currently introduced which consider the presence of one silicon atom as storing bit 1 (or 0 ) and the absence it as storing bit 0 (or 1). These memories do the writing process by removing silicon atoms from the gold lattice. Reading process is performed by nano-tips that can detect the presence or absence of silicon atoms. However, they are not rewritable. Recently, IBM corp. has developed the magnetic atomic memories. In these memories, the single magnetic atoms are located over a surface by using magnetic force thus each atom can be mapped to a single bit. For the time being, the nanomemories are facing with challenges. First, the current nano-memories require complex and expensive devices to be written. For the programmable nano-sensors, the read and write capabilities of memory in nano-scale are necessary. Second, similarly to the nano-processor, the requirement is the technologies to be able to produce very small nano-memories in large number and beyond simplified laboratory prototypes.

- Communication unit: the usage of common frequency ranges from hundreds of $\mathrm{MHz}$ to several $\mathrm{GHz}$ means that the size of antenna will be a few centimeter, which is impossible for nano-scale communication [7]. If the size of classic antenna is reduced to a few hundred of nanometer, should be used of the extremely high operating frequency in $\mathrm{THz}$ band, which caused many problems in electromagnetic communication (such as high attenuation). However, the graphene can be used to build nano-antenna. Therefore, it can overcome this limitation. A graphene nano-antenna with $1 \mu \mathrm{m}$ long can emit the electromagnetic waves within the range 1-10 terahertz band [6]. Moreover, graphene can be used to build an atom-precise antenna. A nano-dipole antenna can be manufactured by a single or a set of carbon nanotube. A single graphene nanoribbon can also be used to build a nano-patch. The communication mechanism of electromagnetic nano-antenna is different from the traditional antenna. In traditional communication the electrons oscillate inside the antenna in response to electromagnetic wave, while in nano-antenna, if the CNTs are charged, they oscillate [7]. The wave propagation 
velocity in nano-antenna based on CNTs or GNRs is significantly less than the speed of light in vacuum (up to one hundred times) [6].

- Electromagnetic nano-transceiver: if a radio wave induces on the nanotube, the charged tip of the nanotube is physically vibrated. When the frequency of incoming wave matches the resonance frequency of nanotube, the nanotube vibration tune to the frequency of wave and thus the nanotube can receive the incoming signal [8]. Electromagnetic transceivers are limited in terms of complexity (space limitation) and integration. They require the RF FET transistors that can support the very high frequencies ( $\mathrm{THz}$ band) [6]. The graphene-based transceivers are faced with several challenges. First, the electronic noise strongly affect the SNR (signal to noise ratio) at the receiver, thus the communication range of nano-device is limited. Therefore, it is necessary to model the electronic noise accurately. Second, the new information modulation techniques must be developed [6].

\subsubsection{Types of nano-sensors}

There are several types of nano-sensors based on different magnitudes that they are able to measure them [6]:

- Physical nano-sensor: this type of nano-sensors can measure the quantities such as mass, pressure, force or displacement. When a CNT or GNR is bent or deformed, its electronic properties change, this is the basis of their functionality. For example, a CNT can be used to build a FET (Field-Effect Transistor) transistor at nano-scale, whose on/off threshold voltage depends on the CNT physical properties such as shape and temperature.

- Chemical nano-sensor: the quantities such as concentration of a specific gas, the presence of specific molecules or a certain molecular composition can be measured by this type of nano-sensors. Their working mechanism is based on changes in the CNT or GNR electrical properties, when different types of molecules are absorbed on top of them. Like to physical nano-sensors, a FET transistor can be built by a nanotube that the presence of a specific type of molecules changes on/off threshold voltage of the transistor.

- Biological nano-sensor: The biological process such as antibody/antigen interaction, DNA interaction and cellular communication process can be monitored by this type of nano-sensors. Their electric properties change when: (a) a protein or other chemical composition is attached to the nanotube (b) a specific antigen is attached to an antibody that located on the nanotube (c) a single-stranded DNA is attached to another DNA chain, which has been connected to the nanotube [6].Fig.14 shows the working principle of these CNT-based nano-sensors.

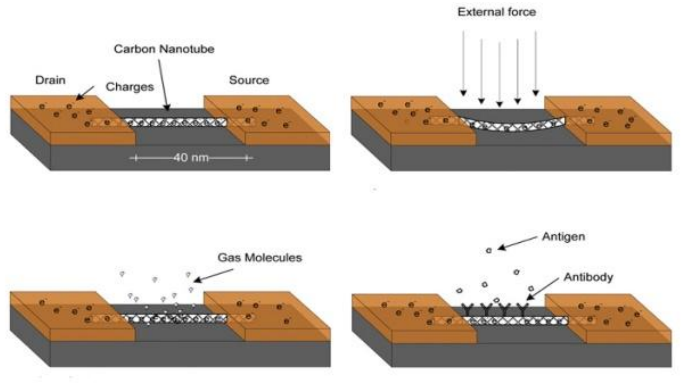

Fig 14: The working principle of physical, chemical and biological nano-sensors [6]

\subsection{Wireless Nano-Network Architecture}

There are the following components in the architecture of electromagnetic nano-networks:

- Nano-nodes: they are able to do simple computation, have limited memory and due to their limited energy and communication constrains, they can only communicate over very short distances. They can also be integrated in objects such as key, book or paper folders.

- Nano-routers: they are greater than nano-nodes in size and have larger computational resources than nano-nodes. They aggregate the information coming from nano-sensor nodes and can issue the simple command to control the behavior of nano-nodes.

- Nano-micro interface: they aggregate the information coming from nano-routers and forward them to a microscale device or vice versa. They are assumed to be hybrid devices, which are able to communicate using nanocommunication techniques and the classical schemes to communicate with traditional networks.

- Gateway: this device can control the nano-sensor network remotely. For example, in health monitoring application, an advanced cellphone can receive the information from a nano-micro interface which is located at wrist and forward data to a healthcare center [6]. Fig.15 shows the Nanonetwork architecture.

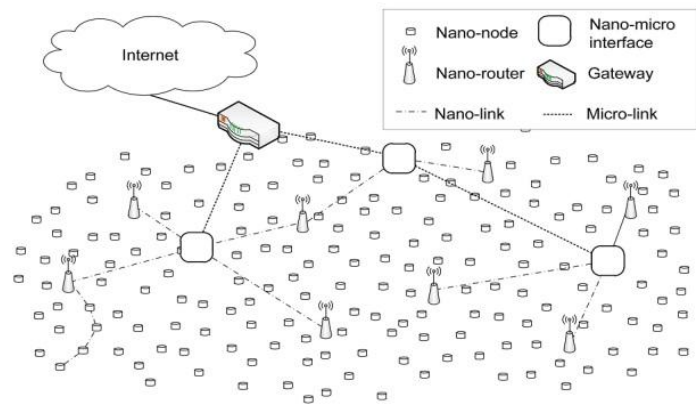

Fig 15: Wireless Nano-Network Architecture [21]

\subsection{Terahertz Channel Properties}

Terahertz channel is seriously affected by the Presence of different molecules in the medium. In addition, high molecular absorption attenuates the travelling wave and introduces noise into the channel. The capacity of channel depends on the molecular composition of the channel and transmission distance. However, that is very large in order of a few terabit per seconds [6]. The main characteristics of the terahertz channel are: 


\subsubsection{Path-loss}

The total path-loss in the terahertz band includes spreading loss and the molecular absorption loss. The expansion of the wave when it propagates through the medium leads to attenuation named spreading loss. The absorption loss is attenuation due to the molecular absorption that leads to a part of wave energy is converted into internal kinetic energy in some of the molecules of the medium [6]. It depends on the concentration and the type of molecular composition of the medium along the path.

\subsubsection{Noise}

The absorption by molecules present in the medium, lead to noise. The total noise in the receiver consists of electronic noise and molecular noise. The predictability of electronic noise is difficult, because of the large free path of electrons in graphene. Therefore, the accurate models are needed. The molecular noise also occurs because of the molecular absorption. This noise is not white or Gaussian noise.

\subsection{Examples of Novel Schemes in Electromagnetic Communication}

Koksal et al. in [7] propose a system that uses multiple CNT-based electromagnetic receivers. The proposed structure consists of a fixed cathode plate and a flexible anode plate on which the CNTs are arranged according to length as shown in fig.16. The electromagnetic bursts that are match with resonance frequencies of CNT lengths can excite the different groups of CNTs with similar lengths. The emission current can differentially change with every electromagnetic wave, and can be mapped to the distance of excited CNT from the cathode. Since the anode flexes, the distance of the CNTs to the cathode can change, and it affects the field of emission current measured at the system. The communication mechanism of this system is different from the traditional antenna. In traditional communication, the electrons oscillate inside the antenna in response to electromagnetic wave, while in this receiver, once the CNTs are charged, they will oscillate. This oscillations cause distance variation of the tip of CNT from the cathode. These variations are known as fluctuations of the emission current. The main property of CNT-based system is that it is possible to communicate in the hundreds of $\mathrm{MHz}$ band with systems, which are hundreds of nanometer in size. They showed that the performance of individual CNT-based antenna is limited that can easily be overcome by using of multiple CNTs in the same receiver system. Finally, they showed that the performance of multiple CNT antennas is better than single CNT antenna.

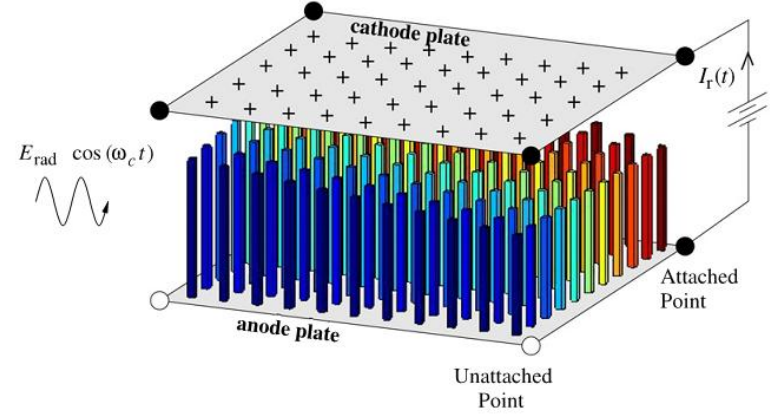

Fig 16: the scheme of system that is composed of multiple CNT-based electromagnetic receivers [7]

Atakan et al. in [8] define the concept of carbon nanotubebased nano-scale Ad hoc networks (CANET). They consider the CANET as a downscaled version of traditional wireless
Ad hoc networks with the same functionality. In CANET, the capabilities of four hardware components i.e. nanotransceiver, nano-power, nano-processor and nano-memory can affect the performance of nano-scale communication among nano-nodes. For this reason, the authors in [8] focus on these nano-scale hardware components and investigate their communication, computation and data storing capabilities for communication in CANET.

\subsection{The Challenges of Nano- Communication Based on Electromagnetic Waves}

Like molecular communication, the electromagnetic communication also has its own challenges such as:

- Communication signals are severely affected by the thermal noise and fading.

- Accurate channel models accounting for molecular absorption, multi-path, molecular noise is required.

- New communication techniques must be presented e.g. sub-picosecond or femtosecond long pulses that are introduced in [10], multicarrier modulations, and MIMO nano-antenna.

- The terahertz band is still not regulated. There is a need to model and develop the future communication standards in $\mathrm{THz}$ for very short-range such as distances much below one meter.

- New information encoding techniques are needed to define the new coding schemes that are suitable for the channel characteristics.

- New MAC and Routing protocols are needed to exploit the properties of the THz band $[6,11]$.

\section{APPLICATIONS OF MOLECULAR AND ELECTROMAGNETIC COMMUNICATION}

Each of communication options has its specific mechanism and properties. According to the intended application, one of the molecular or electromagnetic communications can be useful.

The molecular communication can be used in places that the utilization of electromagnetic waves is not feasible, such as in intra-body applications. Glucose, sodium and cholesterol can be monitored by Nano-machines which are deployed over (e.g. tattoo-like [18]) inside the human body (e.g. a pill or intramuscular injection). Also, they can detect the presence of different infectious agents or cancerous tumor. The potential application of molecular nano-network is in smart drug delivery systems that using of sensing capabilities and nanoactuators to release specific drugs inside the body in a timely manner [6, 17]. Another application of molecular nanomachine is in industrial domain in reaction to biofilms. Depending on the application, the biofilms can be useful or harmful. The biofilms are not often seen by eye. For example, they can be used for cleaning the residual waters coming from manufacturing process, thus they can cause infectious disease. The molecular nano-machines can be used to detect the formation of biofilms and then release specific chemical compounds to destroy their formation [17]. In addition, the molecular nano-network can be used in the environmental applications. For examples, the Nano-machine can be deployed in trees, herbs or bushes and release several 
chemical composites to the air in order to attract the natural predators to deal with insects which are attacking them [6]. Molecular nano-machine can be useful against biological and chemical attacks at the nano-scale. For example, nanomachine can detect toxic chemical composite in a concentration as low as one molecule in a room or in the battlefield $[6,17]$

The electromagnetic nano-network could have applications in Industry, for example, in development of touch surfaces with ultrahigh sensitivity by physical nano-sensors, or in haptic interface application which physical nano-sensors and nanoactuators can enhance the remote control of complex machinery, or in development of an interconnected office which an employee can keep track of all its personal belonging [6]. Also, they can use in damage detection systems, the physical nano-sensor can detect the very small cracks in textile, civil structures, vehicles and even rockets [6].

However, it is possible that both communication options can be used in some applications, but it is clear that just one of them can be the best solution in some specific applications.

\section{CONCLUSION}

This paper has focused on the electromagnetic and molecular communication as promising communication options for nano-devices. We reviewed their communication manners, current communication challenges, applications and novel proposed communication approaches. However, there are many researchers that are working on developing the nanohardware, channel models, modulation techniques and communication protocols such as MAC and Routing protocols for nano-scale network. In summary, the nano-networks are a new communication paradigm which requires fundamental changes in their protocol stack. Therefore, the future researches can continue on issues such as protocol suite, network stacks and channel access procedures that can be applied when the technology is ready for the market.

\section{REFERENCES}

[1] I.F. Akyildiz, F. Brunetti, C. Bl'azquez, "Nanonetworks: A New Communication Paradigm", Elsevier Computer Networks Journal, vol. 52, no. 12, pp. 2260-2279, May 2008.

[2] M. Gregori, I.F. Akyildiz, "A New NanoNetwork Architecture Using Flagellated Bacteria and Catalytic Nanomotors", IEEE Journal on Selected Areas in Communications, vol. 28, no. 4, pp. 612-619, May 2010.

[3] S. Balasubramaniama, N.T. Boyle, A. Della-Chiesa, F. Walsha, A. Mardinoglue, D. Botvicha, A. Prina-Melloc, "Development of Artificial Neuronal Networks for Molecular Communication", Elsevier Nano Communication Networks Journal, vol. 2, no. 2,pp. 150160, June 2011.

[4] A. Enomotoa, M.J. Moore, T. Suda, K. Oiwa, "Design of Self-Organizing Microtubule Networks for Molecular Communication", Elsevier Nano Communication Networks Journal, vol. 2, no. 1, pp. 16-24, March 2011

[5] L.P. Giné, I.F. Akyildiz, "Molecular Communication Options for Long Range Nanonetworks", Elsevier Computer Networks Journal, vol. 53, no. 16, pp. 27532766, November 2009.
[6] I.F. Akyildiz, J.M. Jornet, "Electromagnetic Wireless Nanosensor Networks", Elsevier Nano Communication Networks Journal, vol. 1, no. 1, pp. 3-19, May 2010.

[7] C.E. Koksal, E. Ekici, S. Rajan, "Design and Analysis of Systems Based on RF Receivers with Multiple Carbon Nanotube Antennas", Elsevier Nano Communication Networks Journal, vol. 1, no. 3, pp. 160-172, October 2010.

[8] B. Atakan, O.B. Akan, "Carbon Nanotube-Based Nanoscale Ad Hoc Networks", IEEE Communications Magazine, vol. 48 , no. 6, pp. 129-135, June 2010.

[9] M. Pierobon, I.F. Akyildiz, "A Physical End-to-End Model for Molecular Communication in Nanonetworks", IEEE Journal on Selected Areas in Communications, vol. 28, no. 4, pp. 602-611, May 2010.

[10] J.M. Jornet, J.C. Pujol, J.S. Paret, "PHLAME: A Physical Layer Aware MAC Protocol for Electromagnetic Nanonetworks in the Terahertz Band", Elsevier Nano Communication Networks Journal, vol. 3, no. 1, pp. 74-81, January 2012.

[11] J.M. Jornet, I.F. Akyildiz, "Channel Modeling and Capacity Analysis for Electromagnetic Wireless Nanonetworks in the Terahertz Band", IEEE Transactions On Wireless Communications, vol. 10, no. 10, pp. 3211-3221, October 2011.

[12] H. Sawai, "Biological Functions for Information and Communication Technologies: Theory and Inspiration", chap.2, Springer publisher, January 2011.

[13] M. Gregori, I. Llatsera, A. Cabellos-Aparicioa, E. Alarcon, "Physical Channel Characterization for Medium-Range Nano-Networks Using Flagellated Bacteria”, Elsevier Computer Networks Journal, vol. 55, no. 3, pp. 779-791, February 2011.

[14] M. Gregori, I. Llatsera, A. Cabellos-Aparicioa, E. Alarcon, "Physical Channel Characterization for Medium-Range Nanonetworks Using Catalytic Nanomotors", Elsevier Nano Communication Networks Journal, vol. 1, no. 2, pp. 102-107, June 2010.

[15] T.R. Kline, W.F. Paxton, T.E. Mallouk, A. Sen, "Catalytic Nanomotors: Remote-Controlled Autonomous Movement of Striped Metallic Nanorods", Angewandte Chemie International Edition, vol. 44, pp. 744-746, January 2005

[16] M.S. Kuran, T. Tugcu, B.O. Edis, "Calcium Signaling: Overview and Research Directions of a Molecular Communication Paradigm", Wireless Communications, IEEE Communications Magazine, vol. 19, no. 5, October 2012.

[17] "MoNaCo: Molecular Nano-Communication Networks Project",http://www.ece.gatech.edu/research/labs/bwn/m onaco/projectdescription.html\#SimulationTool, Cited December 2012.

[18] "Northeastern University, Clark Lab", http://nuweb9.neu. edu/nanosensors/?page_id=2, Cited November 2012.

[19] "Nano Networking: a New Frontier in Communications project",http://www.ece.gatech.edu/research/labs/bwn/na nos/projectdescription.html, Cited January 2013. 
[20] "Graphene Used to Create World's Smallest Transistor", http://www.manchester.ac.uk/aboutus/news/display/?id= 3529, Cited November 2012.

[21] J.C. Pujol, "Bridging PHY and MAC Layers in Wireless Electromagnetic Nanonetworks", Final Year Project, Georgia Institute of Technology, December 2010.

[22] "Current Research: Nanonetworks and Molecular Communication", http://www.cse.yorku.ca/ nariman/resea ch.html, Cited January 2013.

[23] "How Bacteria Swim and Tumble", http://www.cellsalive. com/animabug.htm, Cited January 2013. 